\title{
The Associations between Maternal Adverse Childhood Experiences, Substance Use During Pregnancy and Poor Infant Outcomes: A Protocol for Systematic Review and Meta-analysis
}

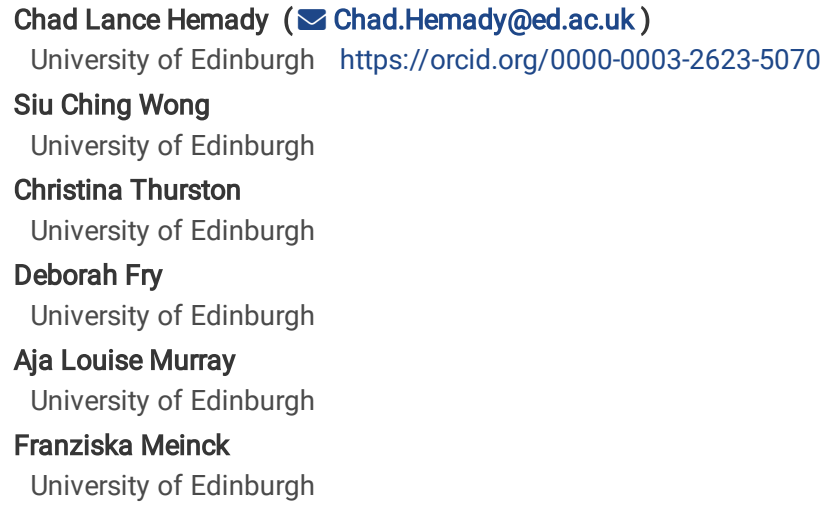

Keywords: adverse childhood experiences, child abuse and neglect, maternal health, early childhood development, infant outcomes, intergenerational transmission of adversity

Posted Date: April 26th, 2021

DOI: https://doi.org/10.21203/rs.3.rs-457143/v1

License: @ (i) This work is licensed under a Creative Commons Attribution 4.0 International License. Read Full License 


\section{Abstract}

Background: Research suggests that maternal exposure to childhood adversity is associated with substance use during pregnancy and poor infant outcomes (i.e., premature birth, low birth weight). However, to date, no systematic review has synthesised the effect of ACEs on these three outcomes.

Method: The framework for this review will be adapted from the guidelines laid out in the Preferred Reporting Items for Systematic Review and Meta-Analysis Protocols (PRISMA-P) 2015 statement. The electronic databases to be searched will include: PubMed, SCOPUS, Psyclnfo, Web of Science, and CINAHL and will be carried out by one reviewer. Studies that fit the pre-specified eligibility criteria will be screened, assessed, and extracted independently by two reviewers with discrepancies to be resolved by a third reviewer. The Newcastle-Ottawa Scale (NOS) for cross-sectional and cohort studies will be used to critically evaluate the methodological quality of the selected studies. If a sufficient number of studies are found to be comparable, a meta-analysis will be conducted using a random effects model.

Discussion: This review will provide supporting evidence on the body of literature exploring the long-term and intergenerational consequences of adverse childhood experiences. The results of the review can help inform policies and interventions related to maternal health as well as early childhood development. The gaps identified in the review can also help inform recommendations for future research.

\section{Introduction}

The Centers for Disease Control and Prevention (CDC) $(2019 ; 7)$ define adverse childhood experiences (ACEs) as 'potentially traumatic events that occur in childhood (0-17 years) such as experiencing violence, abuse, or neglect; witnessing violence in the home; and having a family member attempt or die by suicide'. Its associations with health and development is significant and well documented. ACEs associations with poor health outcomes across the life course include: anxiety/depressive symptoms, post-traumatic stress disorder, borderline personality disorder, suicidality (Angelakis et al., 2019; Dube et al., 2003; Grasso et al., 2016; Herzog \& Schmahl, 2018; Kessler et al., 2005; Widom et al., 2006); obesity and type-2 diabetes (Danese \& Tan, 2014; Thomas et al., 2008); cancer and chronic diseases (Felitti et al., 1998) and premature mortality (D. W. Brown et al., 2009). Moreover, ACEs have been linked with adverse behavioural outcomes such as behavioural disorders (Freeman, 2014), risky sexual behaviours during adolescence (Brown et al., 2015a; Hillis et al., 2004), intimate partner aggression (Brown, et al., 2015b) and substance disorders in adolescence (Dube et al., 2006), adulthood (Dube et al., 2003) , and in pregnancy (Appleyard, Berlin, Rosanbalm, and Dodge, 2011).

Given its significant associations with health and development throughout the lifespan, it is unsurprising that there is growing body of evidence to support that adversity can be transmitted to the subsequent generation (Moog et al., 2016). Specifically, studies suggest a linkage between mothers' experiences of childhood adversity and poor infant outcomes and adverse child development mediated through biological and behavioural pathways (Buss et al., 2017; Madigan et al., 2017). For example, transmission through biological pathways primarily occur through the bilateral effects of maternal ACEs on the maternalplacental-foetal gestational biology that interact during the process of foetal programming. Alterations in the two major biological pathways (endocrine and the immune-inflammatory physiology), affected by ACEs, pour over into gestational biology and cascade into embryonic and foetal development (Moog et al., 2016). On the other hand, behavioural mechanisms such as substance use during pregnancy (Blalock et al., 2011; Widom et al., 2006) are found to mediate maternal exposure to childhood adversity and adverse infant outcomes. For example, a prospective cohort study involving 2,303 pregnant women from the United States found that perinatal smoking and illicit drug use had a mediating effect on maternal adverse childhood experiences and offspring low birth weight and early gestational age (Smith, Gotman, and Yonkers; 2016). Specifically, after adjusting for ethnicity, perinatal smoking accounted for 9.48 grams decrease in birth weight $(\mathrm{Cl}-14.48,-5.67)$ and a 0.020 decrease in weeks in gestational age ( $\mathrm{Cl}-.044,-.007)$ for each unit of increase of ACEs; while illicit drug use accounted for 2.73 grams decrease in birth weight (Cl-5.82, -0.64). Similarly, Gavin, Hill, Hawkins, and Maas (2011) involved three generations of participants $(\mathrm{N}=132)$ from two longitudinal studies (Seattle Social Development Project) and found that antenatal cigarette smoking and heavy alcohol use $(B=-.26, p$-value $=<.001)$ mediated the association between childhood maltreatment and offspring low birth weight. Given the substantial burden of adversity on the individual, it is important to understand the scope of influence maternal experiences of childhood adversity have on their infant's health and development. Although the evidence base on ACEs and its impact on adult health outcomes are well established, there are still limited primary studies which assessed the risk of intergenerational transmission of adversity (Su, D'Arcy, and Meng; 2020).

Through a brief scope of the literature, three systematic reviews were identified which explored the impact of ACEs on preterm birth and low birth weight. A doctoral dissertation by Wiggan (2019) explored the impact of maternal adverse childhood experiences as the exposure and preterm birth as the outcome. The study yielded ten studies (nine from the US and one from Germany), eight of which found a statistically significant relationship between the predictor and the outcome variable. Further, four studies suggest that substance use during pregnancy played a mediating role between the two variables. Similarly, Wosu, Gelaye, and Williams (2015) conducted a systematic review on studies with preterm birth as the outcome variable but limited the predictor variable to maternal history of child sexual abuse. The review yielded six studies and overall, the findings were inconsistent. Of the six (two from the US and one from Germany), only three studies reported a statistically significant correlation between child sexual abuse and preterm birth (See Stevens-Simon 1994; Noll, 2007; and Leeners, 2010). More broadly, Nesari and colleagues (2018) conducted a systematic review with meta-analysis on maternal history of abuse (i.e., at any time in life prior to pregnancy) and its impact on preterm birth and low birth weight. The review included sixteen studies (ten from the US and six from Europe) and found that abuse prior to pregnancy increased the odds of preterm delivery by $1.28(\mathrm{Cl} 1.12-1.47)$ and low birth weight by 1.35 (Cl $1.14-1.59)$. Further, childhood abuse increased the risk of low birth weight by $57 \%(\mathrm{Cl} 0.99-2.49)$.

With a broader scope, three reviews focussed on the impact of parental ACEs on the subsequent generation's development. Su, D'Arcy \& Meng (2020) quantitatively synthesised twelve studies (seven from North America, four from Europe and one from Asia) which explored the intergenerational impact of maternal childhood maltreatment on their children's psychopathology and found that maternal ACEs had small but statistically significant adverse consequence on the child's mental health. The study found that maternal depression and ethnicity moderated relations of the former and the latter suggesting 
that non-Caucasians were more vulnerable to the transmission. A meta-analysis by Madigan and colleagues (2019) which examined 142 studies ( 94 from North America, 26 from Europe, 7 from South America, 7 from New Zealand and Australia and 8 from Africa and Asia) found a significant albeit modest association of intergenerational maltreatment $(\mathrm{d}=.41 \mathrm{Cl} .33-.49)$ suggesting that parents with exposure to childhood maltreatment are more likely to have children who are victimised by maltreatment. Similarly, Greene, Haisley, Wallace, and Ford (2020) quantitatively synthesised 97 studies (6 from Asia, 1 from South America, and 90 from North America and Europe) investigating the impact of parental childhood maltreatment on parenting behaviours (positive and negative) and found that exposure to violence at home had a cumulative effect and increased risk for abusive or neglectful parenting.

Reviews exploring the linkage between maternal childhood adversity and substance use during pregnancy are much sparser. For example, Skagerstróm, Chang, and Nilsen (2011) conducted a review on the predictors of drinking during pregnancy. Three studies out of the fourteen included found that exposure to violence is a predictor of perinatal alcohol consumption; however, the time of abuse prior to pregnancy was not specified in all three studies ( see Harrison \& Sidebottom, 2009; Leonardson, Loudenburg, and Struck, 2007; Meschke, Hellerstedt, Holl, and Messelt 2008). Similarly, conducted a systematic review and meta-analysis on ACEs and associated adult health outcomes. Of the 96 articles included in the review, 13 studies included tobacco use, while 12 and 7 studies explored alcohol problem and illicit drug use as the health outcome, respectively. Pooled unadjusted odds ratio suggests a stepwise gradient between ACEs and the outcomes stated above. Only 2 studies explored the impact of substance use in pregnancy (see Christiaens, Hegadoren, and Olson , 2015; Frankenberger, Clements-Nolle, and Yang 2015). To the best of the reviewers' knowledge, no systematic review has been carried out which synthesised the effects of ACEs on all three outcomes: maternal substance use during pregnancy, and infant low birth weight and prematurity. Additionally, most of the reviews on the outcomes of interest are no longer recent and there is a lot of additional evidence published in the subsequent years given the rise of studies exploring ACEs. This review can also assist in mapping out the aetiology and pathways of ACE-related disease risk which are important factors that help shape prevention and intervention strategies. Moreover, a meta-analysis will allow us to pull together the quantitative results of primary studies and produce a pooled estimate of the associations of the variables of interest that could help inform national and international policies involving child protection, violence prevention, maternal health, and early childhood development. A meta-analysis will also allow us to explore the moderating role of study-level factors such as location of study and publication status and evaluate whether the same inferences can be drawn regardless of publication status or country setting.

\section{Methodology}

The framework for this systematic review will be adapted from the guidelines laid out in the Meta-analysis Of Observational Studies in Epidemiology (MOOSE) (Stroup et al., 2000) as well as the Preferred Reporting Items for Systematic review and Meta-Analysis Protocols (PRISMA-P) 2015 statement the Updating guidance for reporting systematic reviews: development of the PRISMA 2020 statement (Page et al., 2021)This systematic review protocol will be registered on PROSPERO.

\section{Review Question and Objectives}

This systematic review and meta-analysis aims to address the gap in the literature by exploring the interrelationships of maternal exposure to childhood adversity, substance use during pregnancy, and subsequent poor infant outcomes.

Specifically, the research questions are:

i. What is the evidence of the association between ACEs and substance use during pregnancy?

ii. What is the evidence of the association between ACEs and preterm birth?

iii. What is the evidence of the association between ACEs and low birth weight?

iv. Does substance use during pregnancy play a mediating role between maternal ACEs and infant preterm birth and/or low birth weight?

v. Do study-level factors such as publication status, methodological quality, and location of study moderate the effect of these associations?

\section{Definitions:}

The definition of 'children' used in this review follows that on the Conventions on the Right of the Child: 'A child is any person under the age of 18' (UN General Assembly, 1989). The definition of infant follows that of the Centers for Disease Control and Prevention (2021) which are children below 13 months.

'Adverse childhood experiences' used for this review refers to: 'potentially traumatic events that occur in childhood ( 0 - 17 years) such as experiencing violence, abuse, or neglect; witnessing violence in the home; and having a family member attempt or die by suicide. Also included are aspects of the child's environment that can undermine their sense of safety, stability, and bonding such as growing up in a household with substance misuse, mental health problems, or instability due to parental separation or incarceration of a parent, sibling or other member of the household' (CDC, 2019; 7). In addition, this review will include studies related to ACEs that occur outside the household, including exposure to peer violence, community violence, war and collective violence (World Health Organization, 2018). Table 1 exhibits the different types of ACEs included in this review.

By 'substance use during pregnancy' we refer to teratogens consumed at any point during pregnancy that may cause malformations on the embryo. These include: tobacco products, alcoholic beverages, cannabis, cocaine, amphetamine, inhalants, sedatives, hallucinogens, or opioids (WHO ASSIST Working Group, 2002). It is worthy to note that the CDC (2005) cautions against any alcohol consumption due to the significant risks to foetal and later child development.

By 'preterm birth' we follow the broad definition as births with fewer than 37 weeks completed during pregnancy or less than 259 completed days from the mother's last menstrual period (World Health Organization, 2012a). By 'low birth weight' we follow the definition of World Health Organization (2012b) as birth weight less than 2.5 kilograms. 


\begin{tabular}{|ll}
\hline Category & Written Question \\
\hline Physical abuse & Did a parent, guardian or other household member spank, slap, kick, punch or beat you up? \\
\hline Emotional abuse & $\begin{array}{l}\text { Did a parent, guardian or other household member hit or cut you with an object, such as a stick (or } \\
\text { cane), bottle, club, knife, whip etc? }\end{array}$ \\
$\begin{array}{l}\text { Did a parent, guardian or other household member yell, scream or swear at you, insult or humiliate } \\
\text { you? }\end{array}$
\end{tabular}

Did a parent, guardian or other household member threaten to, or actually, abandon you or throw you out of the house?

Sexual Abuse

Did someone touch or fondle you in a sexual way when you did not want them to?

Did someone make you touch their body in a sexual way when you did not want them to?

Did someone attempt oral, anal, or vaginal intercourse with you when you did not want them to?

Did someone actually have oral, anal, or vaginal intercourse with you when you did not want them to?

Alcohol and/or drug abuser in the household

Did you live with a household member who was a problem drinker or alcoholic, or misused street or prescription drugs?

Incarcerated household member

Did you live with a household member who was ever sent to jail or prison?

Someone chronically depressed, mentally ill, institutionalized or suicidal

Did you live with a household member who was depressed, mentally ill or suicidal?

Did you see or hear a parent or household member in your home being slapped, kicked, punched or beaten up?

Household member treated violently

Did you see or hear a parent or household member in your home being yelled at, screamed at, sworn at, insulted or humiliated?

Did you see or hear a parent or household member in your home being hit or cut with an object, such as a stick (or cane), bottle, club, knife, whip, etc.?

One or no parents, parental separation or divorce

Were your parents ever separated or divorced?

Did your mother, father or guardian die?

Emotional neglect

Did your parents/guardians understand your problems and worries?

Did your parents/guardians really know what you were doing with your free time when you were not at school or work?

Physical neglect

Did your parents/guardians not give you enough food even when they could easily have done so?

Were your parents/guardians too drunk or intoxicated by drugs to take care of you?

Did your parents/guardians not send you to school even when it was available?

Bullying

Were you bullied?

Community violence

Did you see or hear someone being beaten up in real life?

Did you see or hear someone being stabbed or shot in real life?

Did you see or hear someone being threatened with a knife or gun in real life?

Collective violence

Were you forced to go and live in another place due to any of these events?

Did you experience the deliberate destruction of your home due to any of these events?

Page 5/12 
Were you beaten up by soldiers, police, militia, or gangs?

Was a family member or friend killed or beaten up by soldiers, police, militia, or gangs?

The elements of the review question are as follows:

Population: mothers who have experienced adversity during the first 18 years of life and their infants (0-1 year of age)

Exposure: maternal ACEs

Comparators: mothers who have no or low experience of childhood adversity

Outcomes of interest: maternal substance use during pregnancy, infant low birth weight, and infant prematurity

Study designs: observational studies (cohort, case-control cross-sectional)

Inclusion Criteria

The review proposes to include studies if:

i. the studies are observational (i.e., cohort, case-control, cross-sectional);

ii. included measurement of maternal exposure to adversity (including any of the categories of ACEs mentioned in the definition section) during childhood (<18 years);

iii. included a measure on substance use during pregnancy

iv. included a measure of offspring prematurity and low birth weight

v. a linkage between maternal ACEs and substance use during pregnancy was assessed

vi. a linkage between maternal ACEs and preterm birth and/or low birth weight was assessed;

vii. the study made use of prospective or retrospective study design to assess associations;

viii. studies conducted between 1998 - 2021[1];

ix. studies are in English

Exclusion Criteria:

The review proposes to exclude:

i. Book chapters, case series studies, case reports, and editorials;

ii. Qualitative studies or studies with no quantitative data;

iii. Studies focussing on pregnancy that occurred during adolescence;

iv. Studies that focus on the biological transmission of adversity (e.g., stress);

v. Studies that focus on maternal exposure to adversity (e.g., abuse, household dysfunction) beyond 18 years of age

Search Strategy

This review will aim to be comprehensive in its approach. It will include both published and unpublished studies which will be identified through computerised searches. Electronic databases to be searched will include: PubMed, SCOPUS, Psycinfo, Web of Science, and CINAHL. Additionally, freely accessible web search engines such as Google Scholar, TRIP database, and Sumsearch2 will be examined. Footnote chasing, forward citation searches, and hand searching non-indexed relevant journals will also be conducted. To limit publication bias, Grey Literature Report, OpenGrey, Virtual Health Library ProQuest Dissertations \& Theses databases will be searched as well as WHO country reports, pre-print servers, conference proceedings, and reports by international \& national governmental organisations. The search process will be carried out by one reviewer $(\mathrm{CH})$.

Search Terms

The search strings for the academic databases for the study are as follows:

Search String

('maternal' OR 'mother* or womn) OR ('childhood' OR 'child*) AND ('advers*' OR 'ACEs' OR 'maltreatment' OR 'violence' OR 'abuse' OR 'household dysfunction' OR 'neglect' OR 'negligent treatment' OR 'bully*' OR 'war' OR 'conflict') AND ('substance' OR 'alcohol' OR 'tobacco' OR 'smoking' OR 'cannabis' OR 'cocaine' OR 'amphetamine' OR 'inhalants' OR 'sedatives' OR 'hallucinogens' OR 'opioids' AND 'during pregnancy' OR ('perinatal' OR 'prenatal') OR ('infant' OR 'neonatal' OR 'offspring') OR ('outcomes' OR 'health') OR ('low birth weight') OR ('preterm birth' or 'prematurity')

Preliminary search was conducted in the proposed databases and the search terms used are outlined in Appendix A which includes more specific search terms relevant to the databases.

Page 6/12 
After carrying out the initial searches, the list of references will be loaded to Covidence a systematic review management software. Before conducting formal review, a calibration exercise will be conducted to pilot and refine the eligibility criteria (Centre for Reviews and Dissemination, 2008). Then, the review will follow a 2-phased screening process. First, titles will be screened, then abstracts will be sifted to determine whether they meet the inclusion criteria of the review. Two reviewers (SCW and CT) will take part in this process. Second, papers that meet the criteria and papers with insufficient information on the abstract will be retrieved and reviewed for full-text screening by three reviewers (CH SCW and CT). Any disagreement related to the inclusion criteria will be resolved by the three reviewers and with guidance from FM DB and ALM. The PRISMA flow diagram will be used to visualise the study selection process to specify the total number of articles found, reviewed, included and excluded and the reasons why.

SCW and CT will extract information which will include: general information (authors, article title, citation, type of publication, year of the study, summary, limitations of the study); study characteristics (sample size, geographical location, research question(s), study design, study inclusion/exclusion criteria); participant characteristics (age, ethnicity, socio-economic status, type of measurement of ACEs); Outcome data/results (unit of analysis, measurement/tool used, statistical techniques used, results of study analysis[substance use during pregnancy, infant premature birth, infant low birth weight], confounders). All extracted information will be shared in an online Excel file.

\section{Assessment of Methodological Quality}

The Newcastle-Ottawa Scale (NOS) for cohort studies and case-control will be used to assess the methodological quality of the studies (Wells et al., 1999). These scales assess three domains: sample selection, comparability of cohorts, and outcomes. A score of 6-8 stars indicate high quality, 4-5 for moderate, and fewer than 4 indicating poor quality. The adapted NOS for cross-sectional studies (Modesti et al., 2016) will be used to assess the methodological quality of cross-sectional studies. This scale assesses the same three domains but has a maximum of 10 stars. Two reviewers (CH and CT) from the team will conduct the assessment of methodological quality concurrently with data extraction.

\section{Evidence Synthesis}

Extracted data will be synthesised using a narrative summary. To address the lack of transparency, which is a common criticism for this approach (DixonWoods, Agarwal, Jones, Young, and Sutton, 2016), the Guidance on the Conduct of Narrative Synthesis in Systematic Reviews (Popay et al., 2006) will be closely followed.

Meta-analysis will be conducted if there are at least 5 studies judged to be sufficiently comparable in conceptual and design terms. Quantitative results from the selected studies will be synthesised using the 'Metafor' package (Viechtbauer, 2010) for R statistical software (R Core Team, 2013). It will be supplemented by a narrative summary to synthesise data not captured in the statistical analyses (Tufanaru et al., 2020). Studies will be pooled into subgroups (e.g., type of design, type of outcomes) and moderator analysis will be conducted to test if the location of the studies moderate the associations between ACEs and the specified health outcomes. Additionally, to investigate if publication status plays a moderating role, statistical tools such as the funnel plot and the trim and fill method will be utilised (Duval, 2005; Sterne \& Egger, 2005). Finally, A random-effects model will be utilised comparing both adjusted and unadjusted effects.

[1] The rationale behind the delimiter on the timeline of studies is that the pioneering ACE study by Felitti and colleagues was first published in 1998 and the succeeding years saw an exponential rise in research on this topic including the advent of relevant theories (e.g., Life Course Theory).

\section{Discussion}

This systematic review protocol maps out the proposed steps to be taken to assess the associations of maternal adverse childhood experiences and substance use during pregnancy as well as poor infant outcomes in the form of low birth weight and infant prematurity. There is currently no systematic review carried out which synthesised the effects of maternal ACEs on all three outcomes. It is the aim of this study to fill that gap. The results of the review can help inform policies and interventions related to maternal health as well as early childhood development. Additionally, it will allow for a more precise estimate of the associations than could be possible using any primary study to date, identify overall quality of evidence in the field and identify specific and common methodological weaknesses, and evidence gaps (i.e., where there are few studies covering a topic). This review could inform future research to advance knowledge in this area.

\section{Declarations}

\section{Funding}

Not Applicable

\section{Competing Interests}

Not Applicable

\section{Authors' contributions}

$\mathrm{CH}$ is the principal researcher for this review and had developed the first draft of the protocol with guidance from all reviewers. $\mathrm{CH}$ will search the databases and extract the studies to COVIDENCE. SCW, and CT will conduct the title and abstract screening while $\mathrm{CH}, \mathrm{CT}$, and SCW will be in charge of full text screening. 
Additionally, SCW and CT will extract the studies, and assess the methodological quality of the selected studies while $\mathrm{CH}$ will analyse and synthesise the data with support from CT and SCW and guidance from FM, DF, and AM. Manuscript will be drafted by CH with comments from CT, SCW, FM, DF, and ALM.

\section{References}

Angelakis, l., Gillespie, E. L., \& Panagioti, M. (2019). Childhood maltreatment and adult suicidality: A comprehensive systematic review with meta-analysis. Psychological Medicine, 49(07), 1057-1078. https://doi.org/10.1017/S0033291718003823

Appleyard, K., Berlin, L. J., Rosanbalm, K. D., \& Dodge, K. A. (2011). Preventing Early Child Maltreatment: Implications from a Longitudinal Study of Maternal Abuse History, Substance Use Problems, and Offspring Victimization. Prevention Science, 12(2), 139-149. https://doi.org/10.1007/s11121-010-0193-2

Blalock, J. A., Nayak, N., Wetter, D. W., Schreindorfer, L., Minnix, J. A., Canul, J., \& Cinciripini, P. M. (2011). The relationship of childhood trauma to nicotine dependence in pregnant smokers. Psychology of Addictive Behaviors, 25(4), 652-663. https://doi.org/10.1037/a0025529

Brown, D. W., Anda, R. F., Tiemeier, H., Felitti, V. J., Edwards, V. J., Croft, J. B., \& Giles, W. H. (2009). Adverse Childhood Experiences and the Risk of Premature Mortality. American Journal of Preventive Medicine, 37(5), 389-396. https://doi.org/10.1016/j.amepre.2009.06.021

Brown, M. J., Masho, S. W., Perera, R. A., Mezuk, B., \& Cohen, S. A. (2015). Sex and sexual orientation disparities in adverse childhood experiences and early age at sexual debut in the United States: Results from a nationally representative sample. Child Abuse \& Neglect, 46, 89-102.

https://doi.org/10.1016/j.chiabu.2015.02.019

Brown, M. J., Perera, R. A., Masho, S. W., Mezuk, B., \& Cohen, S. A. (2015). Adverse childhood experiences and intimate partner aggression in the US: Sex differences and similarities in psychosocial mediation. Social Science \& Medicine (1982), 131, 48-57. https://doi.org/10.1016/j.socscimed.2015.02.044

Buss, C., Entringer, S., Moog, N. K., Toepfer, P., Fair, D. A., Simhan, H. N., Heim, C. M., \& Wadhwa, P. D. (2017). Intergenerational Transmission of Maternal Childhood Maltreatment Exposure: Implications for Fetal Brain Development. Journal of the American Academy of Child \& Adolescent Psychiatry, 56(5), 373382. https://doi.org/10.1016/j.jaac.2017.03.001

Centers for Disease Control and Prevention. (2005). Advisory on Alcohol Use in Pregnancy. https://www.cdc.gov/ncbddd/fasd/documents/sg-advisory508.pdf

Centers for Disease Control and Prevention. (2019). Preventing Adverse Childhood Experiences. Atlanta, GA: National Center for Injury Prevention and Control, Centers for Disease Control and Prevention., 40.

Christiaens, I., Hegadoren, K., \& Olson, D. M. (2015). Adverse childhood experiences are associated with spontaneous preterm birth: A case-control study. BMC Medicine, 13(1), 124. https://doi.org/10.1186/s12916-015-0353-0

Currie, C. L., Sanders, J. L., Swanepoel, L.-M., \& Davies, C. M. (2020). Maternal adverse childhood experiences are associated with binge drinking during pregnancy in a dose-dependent pattern: Findings from the All Our Families cohort. Child Abuse \& Neglect, 101, 104348.

https://doi.org/10.1016/j.chiabu.2019.104348

Danese, A., \& Tan, M. (2014). Childhood maltreatment and obesity: Systematic review and meta-analysis. Molecular Psychiatry, 19(5), 544-554.

https://doi.org/10.1038/mp.2013.54

Dube, S. R., Felitti, V. J., Dong, M., Chapman, D. P., Giles, W. H., \& Anda, R. F. (2003). Childhood Abuse, Neglect, and Household Dysfunction and the Risk of Illicit Drug Use: The Adverse Childhood Experiences Study. PEDIATRICS, 111(3), 564-572. https://doi.org/10.1542/peds.111.3.564

Dube, Shanta R., Miller, J. W., Brown, D. W., Giles, W. H., Felitti, V. J., Dong, M., \& Anda, R. F. (2006). Adverse childhood experiences and the association with ever using alcohol and initiating alcohol use during adolescence. Journal of Adolescent Health, 38(4), 444.e1-444.e10.

https://doi.org/10.1016/j.jadohealth.2005.06.006

Duval, S. (2005). The Trim and Fill Method. In Publication bias in meta-analysis: Prevention, assessment and adjustments (Vol. 72, pp. 269-271). John Wiley \& Sons Ltd, The Atrium, Southern Gate, Chichester, West Sussex P019 8SQ, England. http://link.springer.com/10.1007/s11336-006-1450-y

Felitti, V. J., Anda, R. F., Nordenberg, D., Williamson, D. F., Spitz, A. M., Edwards, V., Koss, M. P., \& Marks, J. S. (1998). Relationship of Childhood Abuse and Household Dysfunction to Many of the Leading Causes of Death in Adults. American Journal of Preventive Medicine, 14(4), $245-258$.

https://doi.org/10.1016/S0749-3797(98)00017-8

Frankenberger, D. J., Clements-Nolle, K., \& Yang, W. (2015). The Association between Adverse Childhood Experiences and Alcohol Use during Pregnancy in a Representative Sample of Adult Women. Women's Health Issues, 25(6), 688-695. https://doi.org/10.1016/j.whi.2015.06.007

Freeman, P. A. C. (2014). Prevalence and Relationship Between Adverse Childhood Experiences and Child Behavior Among Young Children. Infant Mental Health Journal, 35(6), 544-554. https://doi.org/10.1002/imhj.21460

Gavin, A. R., Hill, K. G., Hawkins, J. D., \& Maas, C. (2011). The Role of Maternal Early-Life and Later-Life Risk Factors on Offspring Low Birth Weight: Findings From a Three-Generational Study. Journal of Adolescent Health, 49(2), 166-171. https://doi.org/10.1016/j.jadohealth.2010.11.246 
Grasso, D. J., Dierkhising, C. B., Branson, C. E., Ford, J. D., \& Lee, R. (2016). Developmental Patterns of Adverse Childhood Experiences and Current Symptoms and Impairment in Youth Referred For Trauma-Specific Services. Journal of Abnormal Child Psychology, 44(5), 871-886. https://doi.org/10.1007/s10802-0150086-8

Greene, C. A., Haisley, L., Wallace, C., \& Ford, J. D. (2020). Intergenerational effects of childhood maltreatment: A systematic review of the parenting practices of adult survivors of childhood abuse, neglect, and violence. Clinical Psychology Review, 80, 101891. https://doi.org/10.1016/j.cpr.2020.101891

Group, W. A. W. (2002). The Alcohol, Smoking and Substance Involvement Screening Test (ASSIST): Development, reliability and feasibility. Addiction, 97(9), 1183-1194. https://doi.org/10.1046/j.1360-0443.2002.00185.x

Harrison, P. A., \& Sidebottom, A. C. (2009). Alcohol and Drug Use Before and During Pregnancy: An Examination of Use Patterns and Predictors of Cessation. Maternal and Child Health Journal, 13(3), 386-394. https://doi.org/10.1007/s10995-008-0355-z

Herzog, J. I., \& Schmahl, C. (2018). Adverse Childhood Experiences and the Consequences on Neurobiological, Psychosocial, and Somatic Conditions Across the Lifespan. Frontiers in Psychiatry, 9, 420. https://doi.org/10.3389/fpsyt.2018.00420

Hillis, S. D., Anda, R. F., Dube, S. R., Felitti, V. J., Marchbanks, P. A., \& Marks, J. S. (2004). The Association Between Adverse Childhood Experiences and Adolescent Pregnancy, Long-Term Psychosocial Consequences, and Fetal Death. PEDIATRICS, 113(2), 320-327. https://doi.org/10.1542/peds.113.2.320

Kessler et al. (2005). Lifetime Prevalence and Age-of-Onset Distributions of DSM-IV Disorders in the National Comorbidity Survey Replication. Arch Gen Psychiatry, Vol. 62.

Leonardson, G. R., Loudenburg, R., \& Struck, J. (2007). Factors predictive of alcohol use during pregnancy in three rural states. Behavioral and Brain Functions, 3(1), 8. https://doi.org/10.1186/1744-9081-3-8

Madigan, S., Cyr, C., Eirich, R., Fearon, R. M. P., Ly, A., Rash, C., Poole, J. C., \& Alink, L. R. A. (2019). Testing the cycle of maltreatment hypothesis: Meta-analytic evidence of the intergenerational transmission of child maltreatment. Development and Psychopathology, 31(1), $23-51$.

https://doi.org/10.1017/S0954579418001700

Madigan, S., Wade, M., Plamondon, A., Maguire, J. L., \& Jenkins, J. M. (2017). Maternal Adverse Childhood Experience and Infant Health: Biomedical and Psychosocial Risks as Intermediary Mechanisms. The Journal of Pediatrics, 187, 282-289.e1. https://doi.org/10.1016/j.jpeds.2017.04.052

Meschke, L. L., Hellerstedt, W., Holl, J. A., \& Messelt, S. (2008). Correlates of Prenatal Alcohol Use. Maternal and Child Health Journal, $12(4), 442-451$. https://doi.org/10.1007/s10995-007-0261-9

Modesti, P., Reboldi, F., Cappuccio, C., Agyemang, G., Remuzzi, S., Rapi, S., Perruolo, E., Parati, G., \& ESH Working Group. (2016). Panethnic differences in blood pressure in Europe: A systematic review and meta-analysis. PLoS One, 11 (2016), p. e0147601. https://storage.googleapis.com/plos-corpusprod/10.1371/journal.pone.0147601/1/pone.0147601.s001.pdf?X-Goog-Algorithm=G00G4-RSA-SHA256\&X-Goog-Credential=wombat-sa\%40plosprod.iam.gserviceaccount.com\%2F20210217\%2Fauto\%2Fstorage\%2Fgoog4_request\&X-Goog-Date=20210217T102437Z\&X-Goog-Expires=3600\&X-GoogSignedHeaders=host $\&$ X-Goog-

Signature $=d c 9 c b 4 c 164 f 0 b 6 e a d 66 b b d b 179 e 1 a c 482 c 91$ cb0a634e94f29cdf68f72ce898c0a1a72aa3a8ff5f22eddcb327a381ce6c35ccaebbe6c91f1c6ccddf94a

Moog, N. K., Buss, C., Entringer, S., Shahbaba, B., Gillen, D. L., Hobel, C. J., \& Wadhwa, P. D. (2016). Maternal Exposure to Childhood Trauma Is Associated During Pregnancy With Placental-Fetal Stress Physiology. Biological Psychiatry, 79(10), 831-839. https://doi.org/10.1016/j.biopsych.2015.08.032

Page, M. J., McKenzie, J. E., Bossuyt, P. M., Boutron, I., Hoffmann, T. C., Mulrow, C. D., Shamseer, L., Tetzlaff, J. M., \& Moher, D. (2021). Updating guidance for reporting systematic reviews: Development of the PRISMA 2020 statement. Journal of Clinical Epidemiology, 134, 103-112.

https://doi.org/10.1016/j.jclinepi.2021.02.003

Petruccelli, K., Davis, J., \& Berman, T. (2019). Adverse childhood experiences and associated health outcomes: A systematic review and meta-analysis. Child Abuse \& Neglect, 97, 104127. https://doi.org/10.1016/j.chiabu.2019.104127

Popay, J., Roberts, H., Sowden, A., Petticrew, M., Arai, L., Rodgers, M., \& Britten, N. (2006). Guidance on the Conduct of Narrative Synthesis in Systematic Reviews. In A product from the ESRC methods programme Version 1 (p. 92).

Smith, M. V., Gotman, N., \& Yonkers, K. A. (2016). Early Childhood Adversity and Pregnancy Outcomes. Maternal and Child Health Journal, 20(4), 790-798. https://doi.org/10.1007/s10995-015-1909-5

Spatz Widom, C., Marmorstein, N. R., \& Raskin White, H. (2006). Childhood victimization and illicit drug use in middle adulthood. Psychology of Addictive Behaviors, 20(4), 394-403. https://doi.org/10.1037/0893-164X.20.4.394

Stroup, D. F., Berlin, J. A., Morton, S. C., Olkin, I., Williamson, G. D., Rennie, D., Moher, D., Becker, B. J., Sipe, T. A., Thacker, S. B., \& for the Meta-analysis Of Observational Studies in Epidemiology (MOOSE) Group. (2000). Meta-analysis of Observational Studies in EpidemiologyA Proposal for Reporting. JAMA, 283(15), 2008-2012. https://doi.org/10.1001/jama.283.15.2008 
Su, Y., D'Arcy, C., \& Meng, X. (2020). Intergenerational Effect of Maternal Childhood Maltreatment on Next Generation's Vulnerability to Psychopathology: A Systematic Review With Meta-Analysis. Trauma, Violence, \& Abuse, 1524838020933870. https://doi.org/10.1177/1524838020933870

Thomas, C., Hypponen, E., \& Power, C. (2008). Obesity and Type 2 Diabetes Risk in Midadult Life: The Role of Childhood Adversity. PEDIATRICS, 121(5), e1240-e1249. https://doi.org/10.1542/peds.2007-2403

Tufanaru, C., Munn, Z., Aromataris, E., Campbell, J., \& Hopp, L. (2020). Chapter 3: Systematic Reviews of Effectiveness. In E. Aromataris \& Z. Munn (Eds.), JBI Manual for Evidence Synthesis. JBI. https://doi.org/10.46658/JBIMES-20-04

UN General Assembly. (1989). Convention on the Rights of the Child. https://www.ohchr.org/Documents/Professionallnterest/crc.pdf

Wells, G., Shea, B., O'Connell, D., Peterson, J., Welch, V., Losos, M., \& Tugwell, P. (1999). The Newcastle-Ottawa Scale (NOS) for assessing the quality of nonrandomised studies in meta-analyses. http://www.ohri.ca/programs/clinical_epidemiology/nosgen.pdf

Wiggan, M. (2019). Adverse Childhood Experiences and Preterm Birth: A Systematic Review [Doctoral Dissertation]. University of Maryland.

World Health Organization. (2012a). Born too soon: The global action report on preterm birth.

http://www.who.int/pmnch/media/news/2012/201204\%5Fborntoosoon-report.pdf

World Health Organization. (2012b). Guidelines on Optimal Feeding of Low Birth Weight Infants in Low-and Middle-income Countries. World Health Organization.

World Health Organization. (2018). Adverse Childhood Experiences International Questionnaire.

https://www.who.int/violence_injury_prevention/violence/activities/adverse_childhood_experiences/questionnaire.pdf?ua=1

\section{Appendix A: Search Strategy}

\section{SCOPUS}

2 TITLE-ABS ( "maternal" OR "mother*" OR "wom*n") AND ("advers* childhood experience" OR "adverse childhood event") OR ( "childhood adversity" maltreatment" OR "household dysfunction" OR "neglect" OR "negligent treatment" OR "bully*" OR "war" OR "conflict")

3 TITLE ("maternal" OR "mother*" OR "wom*n") AND \{advers* childhood experience\} OR \{adverse childhood event\} OR ("childhood adversity" OR "c maltreatment" OR "household dysfunction" OR "neglect" OR "negligent treatment" OR "bully*" OR "war" OR "conflict") AND ("smoking" OR "alcohol" OR "tobacco" OR "cannabis" OR "cocaine" OR "amphetamine" OR drug*") AND \{during pregnancy\} OR \{perinatal\} OR \{prenatal\}

4 TITLE ("maternal" OR "mother*" OR "wom*n") AND \{advers* childhood experience\} OR \{adverse childhood event\} OR ("childhood adversity" OR "c maltreatment" OR "household dysfunction" OR "neglect" OR "negligent treatment" OR "bully*" OR "war" OR "conflict") AND \{infant outcomes\} OR birth\} OR \{prematurity\}

\section{PUbMED}

1 ("maternal"[Title/Abstract] OR "mother*"[Title/Abstract] OR "women"[All Fields]) AND ("adverse childhood experience"[Title/Abstract] OR "adverse childhood event"[All Fields])) OR ("childhood adversity"[Title/Abstract] OR "childhood trauma"[Title/Abstract] OR "childhood abuse"[Title/Abstract] OR "child maltreatment"[Title/Abstract] OR "household dysfunction"[Title/Abstract] OR "neglect"[Title/Abstract] OR "negligent treatment"[Title/Abstract] OR "bully*"[Title/Abstract] OR "war"[Title/Abstract] OR "conflict"[Title/Abstract])

2 (("maternal"[Title/Abstract] OR "mother*"[Title/Abstract] OR "women"[All Fields]) AND ("adverse childhood experience"[Title/Abstract] OR "adverse childhood event"[All Fields])) OR ("childhood adversity"[Title/Abstract] OR "childhood trauma"[Title/Abstract] OR "childhood abuse"[Title/Abstract] OR "child maltreatment"[Title/Abstract] OR "household dysfunction"[Title/Abstract] OR "neglect"[Title/Abstract] OR "negligent treatment"[Title/Abstract] OR "bully*"[Title/Abstract] OR "war"[Title/Abstract] OR "conflict"[Title/Abstract])) AND (("smoking" [Title/Abstract] OR "alcohol"[Title/Abstract] OR "tobacco"[Title/Abstract] OR "cannabis"[Title/Abstract] OR "cocaine"[Title/Abstract] OR "amphetamine"[Title/Abstract] OR "inhalants"[Title/Abstract] OR "sedative"[Title/Abstract] OR "hallucinogen"[Title/Abstract] OR "opioid" [Title/Abstract]) AND "during pregnancy"[Title/Abstract]) OR "perinatal"[Title/Abstract] OR "prenatal"[Title/Abstract])

3 (("maternal"[Title/Abstract] OR "mother*"[Title/Abstract] OR "women"[All Fields]) AND ("adverse childhood experience"[Title/Abstract] OR "adverse childhood event"[All Fields])) OR ("childhood adversity"[Title/Abstract] OR "childhood trauma"[Title/Abstract] OR "childhood abuse"[Title/Abstract] OR "child maltreatment"[Title/Abstract] OR "household dysfunction"[Title/Abstract] OR "neglect"[Title/Abstract] OR "negligent treatment"[Title/Abstract] OR "bully*"[Title/Abstract] OR "war"[Title/Abstract] OR "conflict"[Title/Abstract])) AND ("infant outcomes"[Title/Abstract] OR "neonatal outcomes"[Title/Abstract] OR "prematurity"[Title/Abstract] OR "preterm birth"[Title/Abstract] OR "low birth weight"[Title/Abstract] OR "gestation age"[Title/Abstract]) 
(("maternal" or "mother*" or "wom*n") and ("advers* childhood experience" or "adverse childhood event")).mp. or ("childhood adversity" or "childhood trauma" or "childhood abuse" or "child maltreatment" or "household dysfunction" or "neglect" or "negligent treatment" or "bully*" or "war" or "conflict").m_titl. [mp=title, abstract, heading word, table of contents, key concepts, original title, tests \& measures, mesh]

21 AND (("smoking" or "alcohol" or "tobacco" or "cannabis" or "cocaine" or "amphetamine" or "inhalants" or "sedative" or "hallucinogen" or "opioid" or "illicit drug*") and ("during pregnancy" or "perinatal" or "prenatal")).mp. [mp=title, abstract, heading word, table of contents, key concepts, original title, tests \& measures, mesh]

("infant outcome*" or "neonatal outcome*" or "prematurity" or "preterm birth" or "low birth weight" or "gestation age").mp. [mp=title, abstract, heading word, table of contents, key concepts, original title, tests \& measures, mesh]

CINAHL

\begin{tabular}{|c|c|c|c|}
\hline & Search Term & Search Options & Results \\
\hline 1 & $\begin{array}{l}\text { AB ( maternal or mother or "wom*n") AND AB (childhood or child or youth) AND AB ( "advers*" or "ACE*" or } \\
\text { trauma or abuse or maltreatment or "household dysfunction" or neglect or "negligent treatment" or "bully*" or } \\
\text { "war" or "conflict") }\end{array}$ & $\begin{array}{l}\text { Limiters - Gender: Female; } \\
\text { Pregnancy; Language: } \\
\text { English } \\
\text { Expanders - Apply } \\
\text { equivalent subjects } \\
\text { Search modes - Find all } \\
\text { my search terms }\end{array}$ & 10,425 \\
\hline 2 & $\begin{array}{l}\text { AB ( smoking or tobacco or cigarette or nicotine) OR AB ( alcohol or cannabis or cocaine or amphetamine or } \\
\text { inhalants or sedative or hallucinogen or opioid or illicit drug ) AND AB ( "during pregnancy" or "perinatal" or } \\
\text { "prenatal" ) }\end{array}$ & $\begin{array}{l}\text { Expanders - Apply } \\
\text { equivalent subjects } \\
\text { Search modes - Find all } \\
\text { my search terms }\end{array}$ & 84,035 \\
\hline 3 & 1 AND 2 & $\begin{array}{l}\text { Limiters - Gender: Female } \\
\text { Expanders - Apply } \\
\text { equivalent subjects } \\
\text { Search modes - Find all } \\
\text { my search terms }\end{array}$ & 744 \\
\hline 4 & $\begin{array}{l}\text { AB "infant outcome*" or "neonatal outcome*" or "prematurity" or "preterm birth" or "low birth weight" or } \\
\text { "gestation age" }\end{array}$ & $\begin{array}{l}\text { Limiters - Gender: Female } \\
\text { Expanders - Apply } \\
\text { equivalent subjects } \\
\text { Search modes - Find all } \\
\text { my search terms }\end{array}$ & 22,440 \\
\hline & 1 AND 4 & $\begin{array}{l}\text { Limiters - Gender: Female } \\
\text { Expanders - Apply } \\
\text { equivalent subjects } \\
\text { Search modes - Find all } \\
\text { my search terms }\end{array}$ & 483 \\
\hline
\end{tabular}

Search terms for other sites 
(maternal or mother or "wom*n" ) AND ( childhood or child or youth ) AND ( "advers*" or "ACE*" or trauma or abuse or

Limiters -

maltreatment or "household dysfunction" or neglect or "negligent treatment" or "bully*" or "war" or "conflict") AND (smoking or

Gender:

tobacco or cigarette or nicotine ) OR ( alcohol or cannabis or cocaine or amphetamine or inhalants or sedative or hallucinogen or opioid or illicit drug ) AND( "during pregnancy" or "perinatal" or "prenatal" )

Search

modes -

Find all my

search

terms

\section{Web of science}

$1 \quad(\mathrm{AB}=($ maternal or mother or "wom*n" $))$ AND LANGUAGE: (English)

$2 \quad(A B=($ childhood or child or youth $))$ AND LANGUAGE: (English)

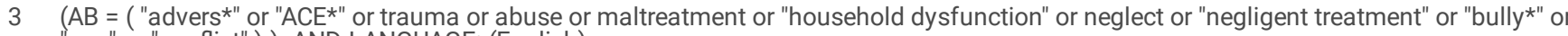
"war" or "conflict" ) ) AND LANGUAGE: (English)

41 AND 2 AND 3 AND ( $\mathrm{AB}=$ ( smoking or tobacco or cigarette or nicotine OR alcohol or cannabis or cocaine or amphetamine or inhalants or sedative or hallucinogen or opioid or illicit drug AND "during pregnancy" or "perinatal" or "prenatal" ) ) AND LANGUAGE: (English)

51 AND 2 AND 3 AND ("infant outcome*" or "neonatal outcome*" or "prematurity" or "preterm birth" or "low birth weight" or "gestation age")

$1 \mathrm{ab}(($ maternal or mother or "wom*n") ) AND ab(( childhood or child or youth ) ) AND ab(( "advers*" or "ACE*" or trauma or abuse or maltreatment or "household dysfunction" or neglect or "negligent treatment" or "bully*" or "war" or "conflict" )) AND ab(( smoking or tobacco or cigarette or nicotine OR alcohol or cannabis or cocaine or amphetamine or inhalants or sedative or hallucinogen or opioid or illicit drug ) ) AND ab(( "during pregnancy" or "perinatal" or "prenatal" ) )

ab((maternal or mother or "wom*n") ) AND ab(( childhood or child or youth ) ) AND ab(( "advers*" or "ACE*" or trauma or abuse or maltreatment or "household dysfunction" or neglect or "negligent treatment" or "bully*" or "war" or "conflict")) AND ab(("infant outcome*" or "neonatal outcome*" or "prematurity" or "preterm birth" or "low birth weight" or "gestation age")

\section{Figures}

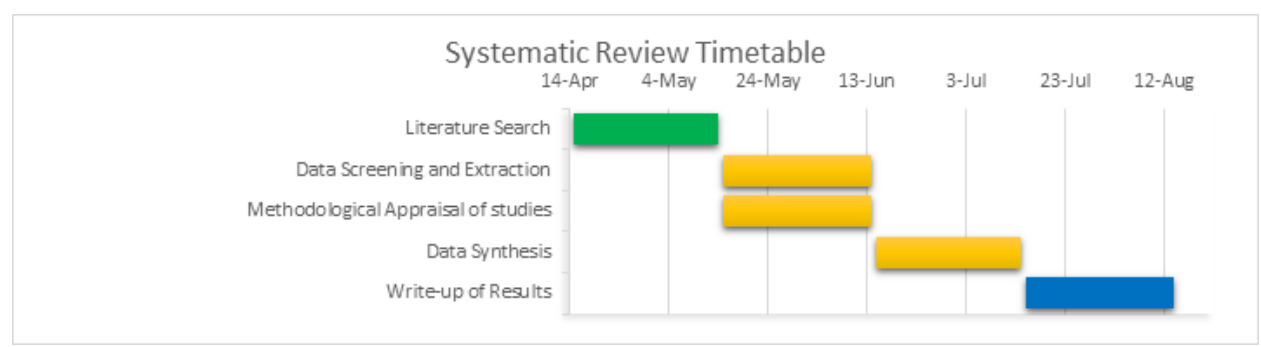

\section{Figure 1}

Systematic Review Timeline 